\title{
DISTRIBUTION PLANNING IN A WEATHER-DEPENDENT SCENARIO WITH STOCHASTIC TRAVEL TIMES: A SIMHEURISTIC APPROACH
}

\author{
Alejandro Estrada-Moreno \\ Marta Cavero-Lazaro \\ Angel A. Juan
}

\author{
Carles Serrat \\ Barcelona School of Building Construction \\ Universitat Politècnica de Catalunya-BarcelonaTECH \\ 08028 Barcelona, SPAIN
}

\author{
Maria Nogal \\ Civil, Structural and Environmental Engineering Department \\ Trinity College Dublin \\ Dublin 2, IRELAND
}

\begin{abstract}
In real-life logistics, distribution plans might be affected by weather conditions (rain, snow, and fog), since they might have a significant effect on traveling times and, therefore, on total distribution costs. In this paper, the distribution problem is modeled as a multi-depot vehicle routing problem with stochastic traveling times. These traveling times are not only stochastic in nature but the specific probability distribution used to model them depends on the particular weather conditions on the delivery day. In order to solve the aforementioned problem, a simheuristic approach combining simulation within a biased-randomized heuristic framework is proposed. As the computational experiments will show, our simulation-optimization algorithm is able to provide high-quality solutions to this NP-hard problem in short computing times even for large-scale instances. From a managerial perspective, such a tool can be very useful in practical applications since it helps to increase the efficiency of the logistics and transportation operations.
\end{abstract}

\section{INTRODUCTION}

The design of effective distribution routes can usually be modeled as a combinatorial optimization problem (COP). In the scientific literature, it has been frequently assumed that these problems were deterministic in nature. However, this assumption is often an unrealistic one that may lead to suboptimal solutions when uncertainty is present. Among other sources of uncertainty, weather conditions might have a significant impact on travel times which, in turn, might affect the total distribution cost. In stochastic COPs, uncertainty is identified with random variables following a probability distribution (Juan et al. 2011a). Stochastic COPs may be solved by designing a solution usable for any specific scenario (aprioristic or robust optimization) or by taking decisions each time uncertain information is revealed (on-line or reactive optimization). When there is complete uncertainty, the last strategy is mostly preferred (Jaillet and Wagner 2008).

In this paper, the distribution problem is modeled as a multi-depot vehicle routing problem (VRP) with stochastic travel times. These travel times are not only stochastic in nature, but the specific probability distribution used to model them depends on the particular weather conditions on the delivery day. Some 
of the earliest works in the field of stochastic vehicle routing problems were based on exact methods (Gendreau et al. 1995), which guarantee the optimality of the solution. However, due to the complexity of these problems, those approaches are only feasible for small-scale instances. In contrast, approaches including heuristics (Dror and Trudeau 1986) and metaheuristics (Bianchi et al. 2006) usually provide near-optimal solutions in reasonable computing times, even for realistic size instances. Accordingly, the stochastic version of the multi-depot VRP considered in this paper is addressed with the use of a simheuristic algorithm. As described in Juan et al. (2015a), a simheuristic is an optimization-simulation framework that combines metaheuristics and simulation (Monte Carlo, discrete-event, agent-based, etc.) to solve a stochastic COP. This hybrid approach has been successfully implemented in several fields, including: Internet computing (Cabrera et al. 2014), transportation (Gonzalez-Martin et al. 2016; de Armas et al. 2017), or production scheduling (Gonzalez-Neira et al. 2017). The main advantages of simheuristics are their flexibility, accuracy, and relatively easy implementation.

Since assignation and routing issues are often interrelated, this problem is a two-stage decision process, where the assignment map will affect the quality of the posterior routing. In the multi-depot VRP with stochastic travel times, a set of customers with known demands must be served by a fleet of homogeneous capacitated vehicles departing from one among several capacitated depots. Moreover, the travel times between each pair of customers, or between each customer and each depot, are random variables following a given probability distribution. These probability distributions might also vary according to the specific weather conditions on the delivery day. In this paper, we will assume that transportation costs will depend upon these travel times. Thus, the main goal of this problem is to determine the customer-to-depot assignment plan and the subsequent routing plan that minimizes the total expected cost of the distribution activity. This optimization process has to take into account the aforementioned constraints on the capacity of each vehicle as well as on the capacity of each depot.

The remainder of this paper is structured as follows: Section 2 motivates the problem considered in this work by providing a real-life case study; in Section 3 some existing work on stochastic versions of the multi-depot VRP is reviewed; Section 4 describes the problem in more detail; our simulation-optimization approach to solve the problem is introduced in Section 5; Section 6 provides a series of computational experiments that contribute to illustrate the efficiency of our approach; and, finally, Section 7 highlights the main conclusions of this work as well as some open research lines.

\section{MOTIVATING THE PROBLEM: THE IRISH CASE}

The reliability of the travel times associated with road transportation depends on a number of factors such as demand fluctuations, traffic incidents, and climate events. Some authors (Lamm et al. 1990; Ibrahim and Hall 1994; Agarwal et al. 2005; Maze et al. 2006; Rakha et al. 2008) have studied on the extent to which the clima impacts the road traffic conditions, reporting that rain, snow, and fog reduce the free-flow speed (up to $43 \%$ in the case of heavy snow), the speed-at-capacity (up to $14 \%$ in the case of heavy rain), and the road capacity (between $10 \%$ and $17 \%$ in the case on rain, and up to $27 \%$ due to snow), resulting in an increment of travel time.

Travel times are usually evaluated by means of congestion functions, i.e., the travel time is considered as a function of the traffic volume in a given road segment. The most common congestion functions (United States 1964; Spiess 1990) also include traffic characteristics, such as road capacity and free-flow speed. Thus, the observed influence of climate upon these traffic characteristics will result in a modification of the travel times obtained by the congestion functions. Additionally, as a consequence of the variation of the travel times in the different roads of the traffic networks, drivers will change their route choices, providing a different map of congestion and, in turn, different travel times. Traffic assignment models derive the congestion maps and the corresponding travel times for different traffic and weather conditions. Thus, the probabilistic distribution of travel times can be obtained by means of traffic assignment models and link congestion functions considering the impact of weather upon the traffic characteristics, which is the most practical way. Nevertheless, the probabilistic distributions can also be based on recorded data. 
The economic and social consequences of this increment are already a source of concern for stakeholders and policymakers, and might become even more important when considering the effect of climate change. We can talk about direct and indirect costs caused by larger travel times. The direct ones refer to the loss of productivity, and within the indirect ones, larger fuel consumption with the corresponding health and environmental impact can be highlighted (Tagliapietra and Zachmann 2018).

According to IPCC (2014), climate change is forecasted to bring more intense weather events more frequently. For instance, events of high-intensity precipitation are expected to increase across Europe while the number of events of heavy snowfall will increase in northern Europe (Nogal et al. 2018).

In the case of Ireland, climate change is expected to notably increase the frequency of heavy precipitation events during winter, up to a significant $20 \%$ during this century (Gleeson et al. 2013). This might worsen the existing costs and delays due to climate-related events (e.g., Brazil et al. 2017). For instance, in February 2018, 5 days of snow-related traffic disruptions all over the country caused losses for the transport companies estimated at more than $160 \mathrm{~m}$ Euro. As a consequence, most of the stores and retailers had to close because of the problems with the goods supply.

The adaptation to weather-related impacts the land-based transport networks is identified as a key strategy to minimize the consequences of climate change (Nogal et al. 2016). This paper introduces the consideration of the weather events when planning the transportation logistics, given that the optimal planning associated with good climate conditions might be far from the actual optimal choice under disruptive climate events. Thus, modifying the logistics according to the weather warnings provided by the meteorological agencies seems a realistic and practical way of adaptation to climate change.

For instance, the Irish Meteorological service Met Eireann classifies the weather warnings into Green, Yellow, Orange, and Red in alignment with the common European framework. Each warning level has associated specific weather characteristics; e.g., the orange warning status due to rain is related to rainfalls of $50 \mathrm{~mm}$ to $70 \mathrm{~mm}$ in $24 \mathrm{hrs}$, or $40 \mathrm{~mm}$ to $50 \mathrm{~mm}$ in $12 \mathrm{hrs}$, or $30 \mathrm{~mm}$ to $40 \mathrm{~mm}$ in $6 \mathrm{hrs}$. Therefore, it is possible to infer the traffic conditions in stochastic terms of speed and capacity associated with each weather warning status, allowing the estimation of the corresponding travel times and costs.

\section{RELATED WORK}

This section reviews related work on two of the main topics discussed in this paper: (i) stochastic multi-depot VRPs; and (ii) simheuristic approaches in logistics and transportation.

\subsection{The Stochastic Multi-Depot VRP}

To the best of our knowledge, only a limited number of works have addressed stochastic versions of the multi-depot VRP. Thus, for instance, Tillman (1969) expands a well-known heuristic to address it. The procedure proposed may be applied to demands with Poisson, exponential, normal, binomial or chi-squared distributions. In Chan et al. (2001), a location routing problem with stochastic demands is analyzed. The random demands are estimated in advance, before the vehicle location-routing decisions are made. Tatarakis and Minis (2009) study the stochastic multi-depot VRP considering both the case in which products are stored dedicatedly or together in a compartment. Dynamic programming algorithms are proposed to determine the minimal routing cost, and an optimal routing policy is derived to decide whether a vehicle has to return to the depot for a reload after serving the current customer or should continue to the next customer. In Zuhori et al. (2012), the authors solve the stochastic multi-depot VRP in three phases: first a nearest neighbor classification method is used for grouping the customers; then, the sum-of-subsets method is applied for routing; and finally, the routes are optimized via a greedy heuristic. They aim at minimizing the number of routes and, accordingly, the number of vehicles needed. None of the aforementioned works deals with the multi-depot VRP with stochastic times and considering capacitated depots, which is the realistic scenario considered in our work. 


\subsection{Simheuristic Approaches to Solve Stochastic COPs}

Simheuristics have been used to solve stochastic COPs that arise in different fields. The first application of simheuristics was in the area of VRPs. Thus, Juan et al. (2011a) consider a vehicle-routing problem with stochastic demands and design a basic simheuristic approach to solve this problem. The authors continue the study of the stochastic VRP in Juan et al. (2013) with the analysis of the parallel and distributed computing techniques. Juan et al. (2014b) consider the stochastic version of an inventory routing problem with stock-outs and design a simheuristic to cope with this hybrid inventory and vehicle routing problem. Juan et al. (2014a), on the other hand, propose a simheuristic algorithm for solving the permutation flow-shop problem with stochastic processing times. An example of a simheuristic application for solving the arc-routing problem with stochastic demands is discussed in Gonzalez-Martin et al. (2016). Another interesting application of the simheuristic approach is in the area of waste collection management. Gruler et al. (2017) consider the stochastic waste-collection problem with a single-depot. de Armas et al. (2017) analyze the stochastic uncapacitated facility location problem with a simheuristic approach. Pagès-Bernaus et al. (2017) consider the problem of designing e-commerce supply chains and propose a simheuristic approach for the stochastic capaciated facility location problem that arises in this context. Very recently, Gruler et al. (2018) tackle a stochastic multi-period inventory routing problem with a variable neighborhood search simheuristic and Panadero et al. (2018) use the same approach in the context of selecting a portfolio of projects under uncertainty.

\section{THE MULTI-DEPOT VRP WITH STOCHASTIC TRAVEL TIMES}

In the multi-depot VRP under a weather-dependent scenario, travel times are assumed to be random variables following specific probability distributions. Thus, let $G=\{V, E\}$ be a undirected graph, where $V=V_{f} \cup V_{c}$ is the vertex set including the depots or facilities $\left(V_{f}\right)$ and the customers $\left(V_{c}\right)$, and $E=\left\{\{u, v\}: u, v \in V_{c}, u \neq\right.$ $v\} \cup\left\{\{u, v\}: u \in V_{c}, v \in V_{f}\right\}$ is the edge set connecting vertices in $V$. Each customer $i \in V_{c}$ has a positive demand $d_{i}>0$ that has to be serviced. Each depot $f \in V_{f}$ has assigned a maximum number of vehicles, $m_{f}$, and has no demand. All vehicles are supposed to have the same capacity $Q$, with $Q>>\max \left\{d_{i}\right\}$. Thus, the service capacity of a depot is limited by $m_{f} \cdot Q$. Traveling each edge in $E$ has associated a travel time based cost $T_{i j}=T_{j i}>0$, which is a random variable. The probability distribution (or its specific parametrization) that each time-based cost follows depends upon the weather conditions. A solution to this problem is a set of customer-to-facilities maps and the related plans of round-trip routes departing from each depot that covers all customer demands while satisfying all the capacity constraints (both the ones related to the depots as well as the ones related to the vehicles). As in most VRPs, it is assumed that each customer can only be visited once by a single vehicle.

Concerning the travel time random variable modeling, log-normal distributions have been used by a number of authors to model stochastic travel times (Kaparias et al. 2008, Rakha et al. 2010). The main reason is, as Castillo et al. (2014) note, that this distribution family allows for modeling of positively skewed data, which is an important characteristic to take into account. Moreover, Castillo et al. (2014) study conditions for stability with respect to minimum and maximum operators and they recommend that, despite log-normal family models are not stable with respect to location changes, they refer to scale changes, and are, therefore, appropriate for travel times.

The main goal in this case is to find a feasible solution that minimizes the total expected travel time costs, while satisfying the customer demands and the capacity constraints. Even in its deterministic version, this problem represents a challenge since it integrates a combinatorial assignment problem - in which each customer is assigned to one facility - with several VRPs, one per facility. By also including the stochastic component, the problem becomes even more difficult to solve, which justifies the use of a simulation-optimization approach as the one introduced in this paper. Figure 1 shows a possible solution for a particular instance. 


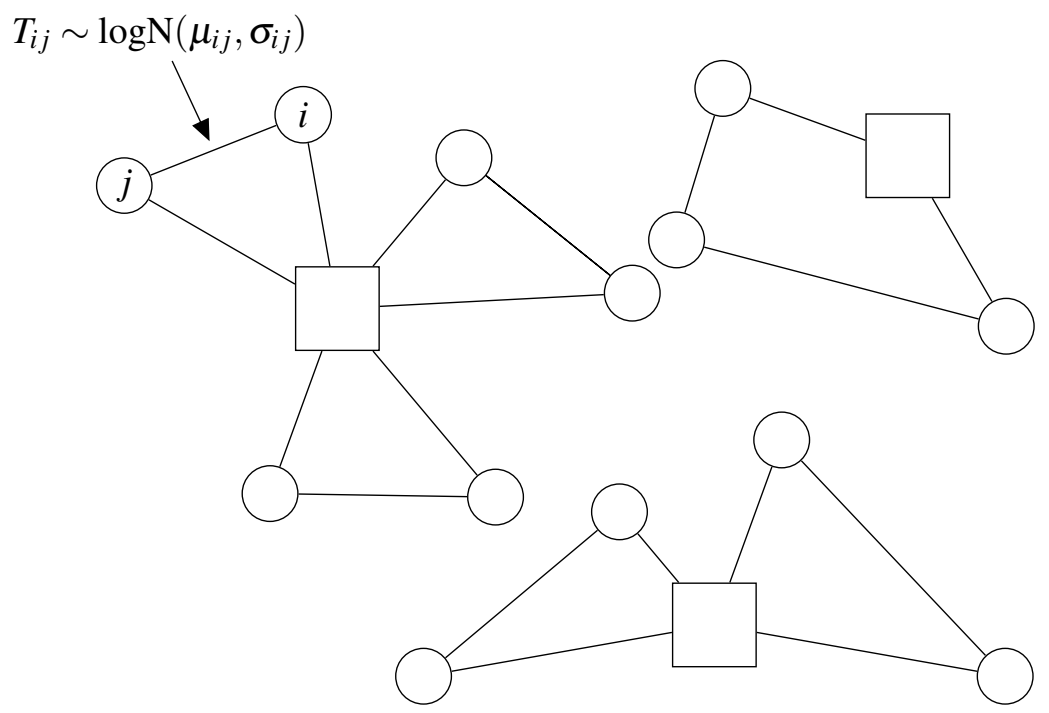

Figure 1: Possible solution for multi-depot VRP with three depots depicted by squares and several customers represented by circles. Travel times are modeled with log-normal distributions.

\section{OUR SIMHEURISTIC APPROACH}

Our approach relies on two facts: (i) the multi-depot VRP with stochastic travel times can be considered a generalization of the multi-depot VRP, i.e., the latter can be seen as a particular case of the former whenever the random demands have zero variance; and (ii) despite the fact that the stochastic version has never been studied before, there exist efficient algorithms for solving the deterministic one. The general ideas behind our approach, which relies on a metaheuristic framework, are described next. Initially, given an instance of the multi-depot VRP with stochastic travel times, it is transformed into a deterministic instance by replacing each random travel time by its expected value. A set of high-quality solutions for the deterministic version is then obtained by using an efficient metaheuristic algorithm that combines biased randomization with iterated local search (Juan et al. 2015b). While the search takes place, Monte Carlo simulation is employed to assess the performance of these promising solutions for the stochastic version. We define the best solution as the one with the lowest total expected travel-time based cost. This assessment is carried out according to the following steps: $(i)$ run hundreds of executions (until the stopping criteria are reached), where each execution implies the generation of random values for each stochastic travel time according to the associated probability distribution, which in turn will depend upon the specific weather conditions; (ii) assess the performance of each solution by estimating the total expected time-based cost as the average of the total time-based costs obtained at the end of each execution; and (iii) use the simulation feedback to better guide the searching process inside the metaheuristic. Figure 2 shows the flow chart of the complete algorithm. In the first stage of the algorithm, an iterated local search (ILS) (Lourenço et al. 2010) is proposed for the generation of new maps. Given that it is critical to evaluate as many assignment maps as possible in the available computing time, every time a map is generated, its deterministic routing cost is estimated by using the well-known savings heuristic (Clarke and Wright 1964). Each solution considered promising from the deterministic point of view passes to a fast simulation process (i.e., low number of replications) to estimate its stochastic cost. Later it is considered whether this solution is ranked as elite solution considering its stochastic cost. This process is repeated until the stopping criterion is reached. Once the "Interaction between metaheuristic driven search and simulation" phase is done, an intensive refinement step is performed. For each of the elite maps obtained in the first stage, a more intensive routing algorithm is applied. Of course, the number of elite maps to consider will depend on the available time and computing resources. The vehicle routing algorithm employed in our tests is the SR-GCWS-CS proposed 
by Juan et al. (2011b). It encapsulates a biased-randomized version of the popular savings heuristic into a multi-start process, which is noticeably enhanced by the use of a cache memory and a 'splitting' technique. A more intensive simulation process is applied for each solution obtained for the deterministic version. A local search is applied, in which from the current assignment customers-depot, it is tried to reassign to a depot those customers who are closest to the customers already assigned. If the stochastic cost of this new customers-depot assignment improves after the simulation and routing process, the local search process is performed. This process is repeated as long as there is improvement in the stochastic cost. Finally, an analysis of risk and reliability is conducted over the elite solutions obtained in this second stage.

\section{COMPUTATIONAL EXPERIMENTS AND DISCUSSION}

The methodology described in this paper has been implemented as a Java application. All the experiments have been run in an Intel Xeon E5-2630 v4 CPU at $2.20 \mathrm{GHz}$ and $32 \mathrm{~GB}$ RAM. For our problem, no benchmark instances exist. Therefore, we adapted first five instances of well-known sets of benchmark instances from the MDVRP described in Cordeau et al. (1997) (instances p01 to p05). In these instances, we have assumed that each distance unit is equivalent to each time unit and, as a consequence, the total distance cost is the total time cost.

In our experiments, in order to model the stochasticity, values for the distances (i.e., times) between customers and customer-depots in each deterministic benchmark have been assumed as the expectation for the travel time density distribution under a log-normality assumption. The scale parameter, in this illustrative approach and without loss of generality, has been assumed as constant (equivalent to assume a constant coefficient of variance) and equal to 0.05 . That is, the travel time distribution between customers $i$ and $j$ is setup as $T_{i j} \sim \log \mathrm{N}\left(\mu_{i j}, \sigma_{i j}\right)$ with $E\left[T_{i j}\right]=\exp \left(\mu_{i j}+\sigma_{i j}^{2} / 2\right)=d_{i j}$ and $\operatorname{Var}\left[T_{i j}\right]=\left(\exp \left(\sigma_{i j}^{2}\right)-1\right) d_{i j}^{2}$, where $d_{i j}$ is the deterministic travel time between both nodes. In practice, the scale parameter $\sigma_{i j}$ was setup in 0.05 .

Stochastic distributional departures due to weather-related conditions have been considered by extending the previous model for the good weather conditions to the one for condition $k,(k \in \mathbb{R}), T_{i j}^{k)}$, as a log-normal density distribution with expectation equal to $d_{i j}(1+k)$ and variance $\left(\exp \left(0.05^{2}\right)-1\right)\left(d_{i j}(1+k)\right)^{2}$. It is straightforward to see that case $k=0$ corresponds to the good weather condition. Notice that, on one hand, when the value of $k$ increases, the weather conditions become worse in mean and in variance and, on the other hand, $E\left[T_{i j}^{k)}\right] \rightarrow E\left[T_{i j}^{0)}\right]=d_{i j}$ and $\operatorname{Var}\left[T_{i j}^{k)}\right] \rightarrow \operatorname{Var}\left[T_{i j}^{0)}\right]$ when $k \rightarrow 0$.

With this configuration, we performed 100 simulations of the minimal expected cost, per each one of the instances and for five weather conditions which are represented by different values of $k,(k=$ $0,0.25,0.5,0.75,1)$.

Under the good-weather scenario (i.e., $k=0$ ), Figure 3 shows the estimation of the kernel density for the distribution of the simulated minimum expected cost for the instance p01, for 100, 1,000, and 10,000 simulations. On one hand, it is noticeable that minimum reported values are really close to the best known solution for the deterministic case. From the practical point of view, it is important to note that around one forth of the total simulated minimum expected costs are located close to the baseline minimal cost (BLC). This proportion is a guide for choosing the appropriate number of simulations to be performed. This is a potential consequence of the proposed algorithm, which shows a relation between the solutions for the stochastic version and for the deterministic version. In other words, the best solutions for the deterministic version are likely to be also high-quality solutions for the stochastic version. Even more, the relation is expected to be stronger as the travel time variances tend to zero. On the other hand, it is also interesting to observe the dependency on the seed values and the multimodal distribution of the results, which might imply that a high number of simulations are needed in order to properly estimate the minimum expected cost in the left tail of the distribution.

Regarding the assessment of the cost increments due to worse weather conditions, Table 1 displays the relative increments (in percentage) with respect to the baseline minimal cost derived from the best known 


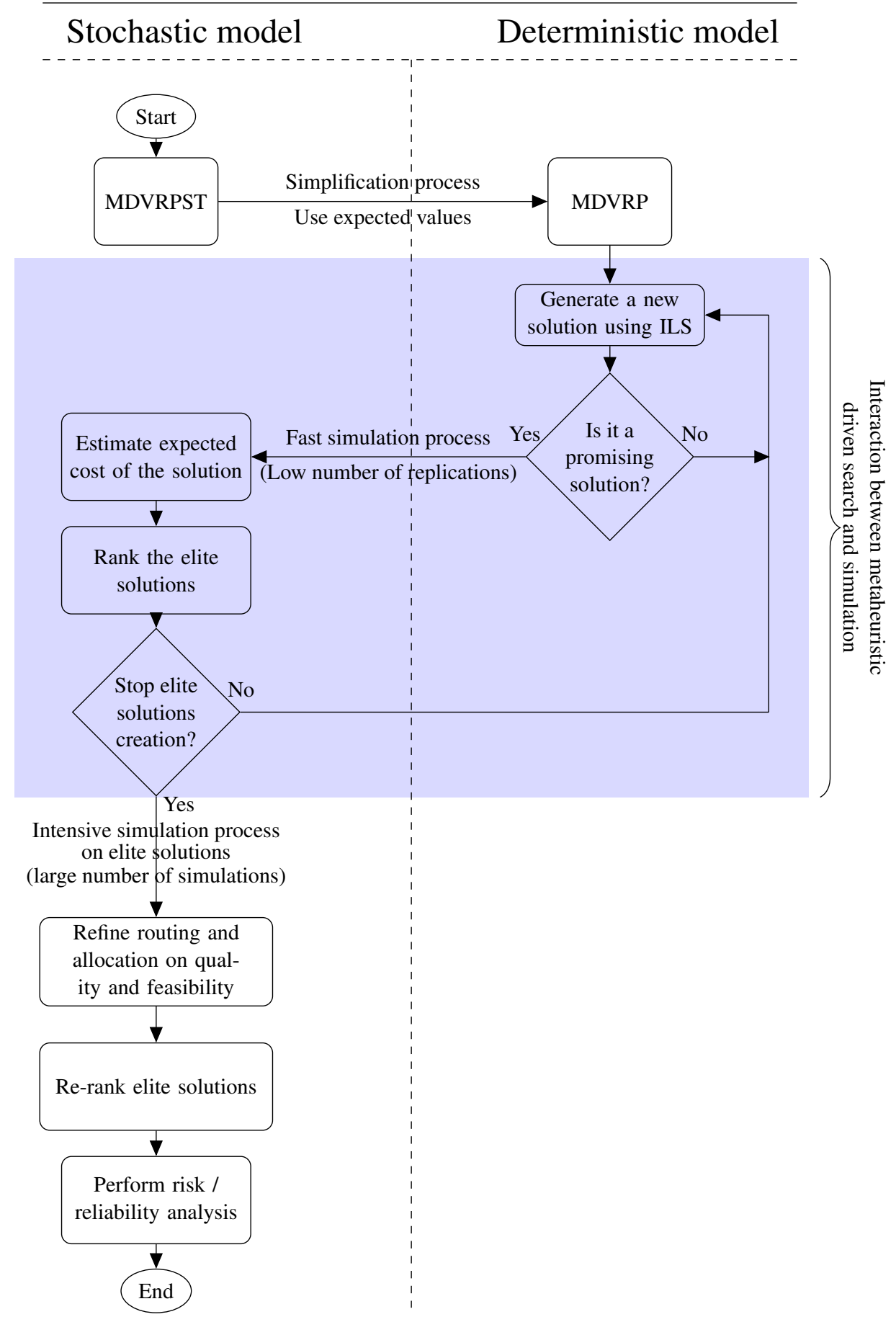

Figure 2: Flow chart of the simheuristics proposal. 


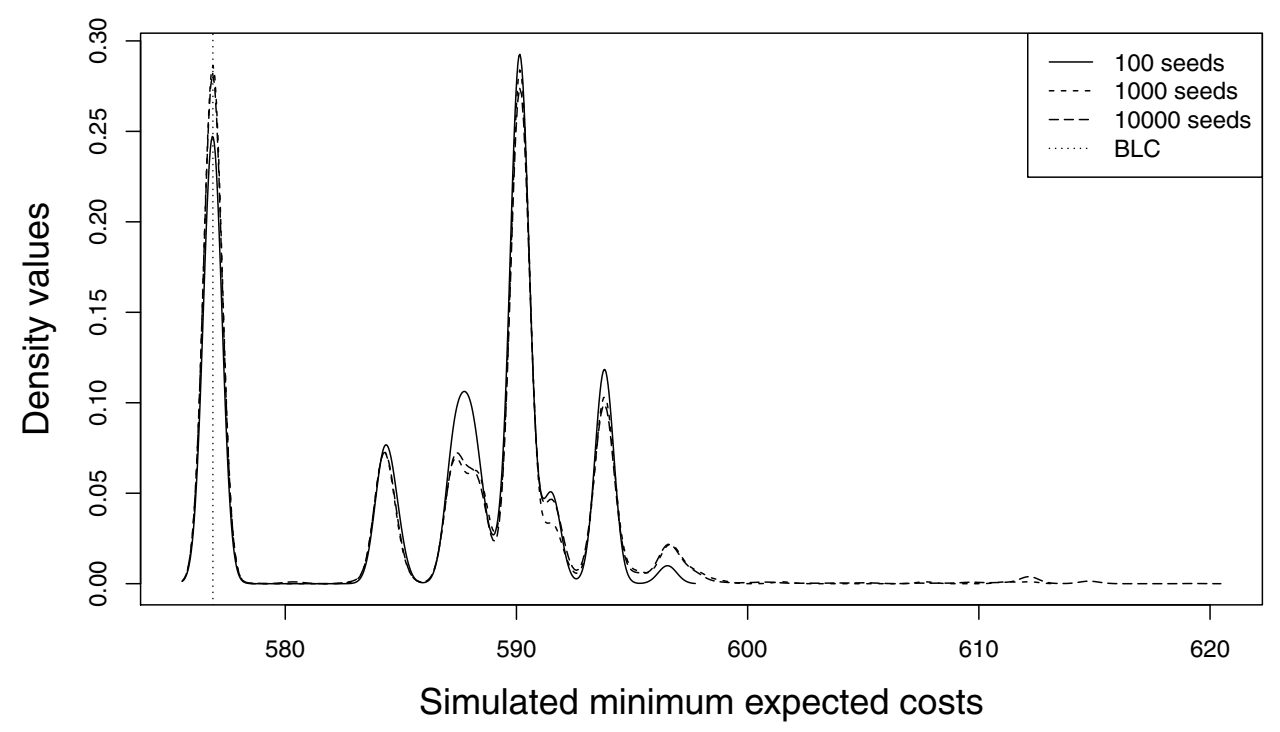

Figure 3: Estimated kernel density for the distribution of the simulated minimum expected costs for the instance p01 with $100,1,000$, and 10,000 seeds and $k=0$. The dotted vertical line corresponds to the best known solution under the deterministic scenario (BLC).

solution for the deterministic case. It is interesting to note that no matter the complexity of the instance, the dilatation coefficient induced by $k$ values is translated in terms of minimum expected cost increments. This a natural consequence of the homothetic global $k$-effect transformation of the stochastic distribution of travel times. In a managerial approach, in a more realistic scenario, a weighted result is expectable, under a different weather condition in each one of the edges of the network.

Table 1: Results for different weather conditions described by $k$ values and applied to 5 instances (p01 to p05). For each instance, characteristics of the instance, BLC and relative increments (in percentage) with respect to BLC are given.

\begin{tabular}{lrrrrr}
\hline PInstance & p01 & p02 & p03 & p04 & p05 \\
\hline \# customers & 50 & 50 & 75 & 100 & 100 \\
\# vehicles & 4 & 2 & 3 & 8 & 5 \\
vehicle capacity & 80 & 160 & 140 & 100 & 200 \\
\# depots & 4 & 4 & 5 & 2 & 2 \\
BLC & 576.87 & 473.53 & 641.19 & $1,001.04$ & 750.03 \\
\hline$k=0$ & -0.01 & -0.04 & -0.01 & 0.26 & 0.23 \\
$k=0.25$ & 24.98 & 24.90 & 24.98 & 25.33 & 25.29 \\
$k=0.5$ & 49.98 & 49.94 & 49.98 & 50.40 & 50.35 \\
$k=0.75$ & 74.97 & 75.05 & 74.98 & 75.46 & 75.41 \\
$k=1$ & 99.97 & 99.92 & 99.97 & 100.53 & 100.47 \\
\hline
\end{tabular}




\section{CONCLUSIONS AND FUTURE WORK}

In this paper, we have addressed the basic strategy for solving an Irish-based realistic scenario, in which the distribution plans need to take into account weather conditions. The scenario has been modeled as a capacitated multi-depot vehicle routing problem with stochastic travel times. As the distribution costs are based on these travel times, our goal is to minimize the total expected time-based cost while satisfying all customer demands and capacity constraints. In order to address this complex problem, a simheuristic algorithm has been proposed. Our approach relies on the combination of a well-tested metaheuristic for solving the deterministic version of the multi-depot vehicle routing problem with Monte Carlo simulation. Simulation is employed not only to assess the quality of the solutions provided by the metaheuristic in a stochastic environment, but also to provide feedback to the metaheuristic in order to make the searching process more efficient. In addition, simulation can also provide valuable data to complete a risk analysis for each of the 'elite' solutions found by the simheuristic algorithm.

Some of the most promising research lines that this work opens include the heterogeneity of the weather effect of the network, as well as the possibility of extending the problem to a multi-period one, where weather forecasts and alerts are used to decide about the actual demands to be served to each customer at any given day of the period as well as to decide about the specific depot that has to cover each customer on that day.

\section{ACKNOWLEDGEMENTS}

This work has been partially supported by the Spanish Ministry of Economy and Competitiveness and FEDER (TRA2015-71883-REDT, MTM2015-64465-C2-1-R), and the Erasmus+ programme (2016-1-ES01KA108-023465).

\section{REFERENCES}

Agarwal, M., T. H. Maze, and R. Souleyrette. 2005. "Impacts of Weather on Urban Freeway Traffic Flow Characteristics and Facility Capacity". In Proceedings of the 2005 Mid-Continent Transportation Research Symposium, August $18^{\text {th }}-19^{\text {th }}$, Iowa State University.

Bianchi, L., M. Birattari, M. Chiarandini, M. Manfrin, M. Mastrolilli, L. Paquete, O. Rossi-Doria, and T. Schiavinotto. 2006. "Hybrid Metaheuristics for the Vehicle Routing Problem with Stochastic Demands". Journal of Mathematical Modelling and Algorithms 5(1):91-110.

Brazil, W., A. White, M. Nogal, B. Caulfield, A. O'Connor, and C. Morton. 2017. "Weather and Rail Delays: Analysis of Metropolitan Rail in Dublin". Journal of Transport Geography 59:69-76.

Cabrera, G., A. Juan, D. Lázaro, J. M. Marquès, and I. Proskurnia. 2014. "A Simulation-Optimization Approach to Deploy Internet Services in Large-scale Systems with User-provided Resources”. Simulation: Transactions of the Society for Modeling and Simulation International 90(6):644-659.

Castillo, E., A. Calvino, M. Nogal, and H. K. Lo. 2014. "On the Probabilistic and Physical Consistency of Traffic Random Variables and Models". Computer-Aided Civil and Infrastructure Engineering 29(7):496-517.

Chan, Y., W. B. Carter, and M. D. Burnes. 2001. "A Multiple-Depot, Multiple-Vehicle, Location-Routing Problem with Stochastically Processed Demands". Computers \& Operations Research 28(8):803-826.

Clarke, G., and J. W. Wright. 1964. "Scheduling of Vehicles from a Central Depot to a Number of Delivery Points". Operations research 12(4):568-581.

Cordeau, J.-F., M. Gendreau, and G. Laporte. 1997. "A Tabu Search Heuristic for Periodic and Multi-Depot Vehicle Routing Problems". Networks 30(2):105-119.

de Armas, J., A. A. Juan, J. M. Marquès, and J. P. Pedroso. 2017. "Solving the Deterministic and Stochastic Uncapacitated Facility Location Problem: from a Heuristic to a Simheuristic". Journal of the Operational Research Society 68(10):1161-1176. 
Dror, M., and P. Trudeau. 1986. "Stochastic Vehicle Routing with Modified Savings Algorithms". European Journal of Operational Research 23(2):228-235.

Gendreau, M., G. Laporte, and R. Séguin. 1995. "An Exact Algorithm for the Vehicle Routing Problem with Stochastic Demands and Customers". Transportation Science 29(2):143-155.

Gleeson, E., R. McGrath, and M. Treanor. (Eds.) 2013. Ireland's Climate: The Road Ahead. Dublin: Met Éireann.

Gonzalez-Martin, S., A. A. Juan, D. Riera, M. G. Elizondo, and J. J. Ramos. 2016. "A Simheuristic Algorithm for Solving the Arc-Routing Problem with Stochastic Demands". Journal of Simulation 12(1):53-66.

Gonzalez-Neira, E. M., D. Ferone, S. Hatami, and A. A. Juan. 2017. "A Biased-randomized Simheuristic for the Distributed Assembly Permutation Flowshop Problem with Stochastic Processing Times". Simulation Modelling Practice and Theory 79:23-36.

Gruler, A., C. L. Quintero-Araújo, L. Calvet, and A. A. Juan. 2017. "Waste Collection under Uncertainty: A Simheuristic Based on Variable Neighbourhood Search". European Journal of Industrial Engineering 11(2):228-255.

Gruler, A., J. Panadero, J. de Armas, J. A. Moreno Pérez, and A. A. Juan. 2018. "A Variable Neighborhood Search Simheuristic for the Multiperiod Inventory Routing Problem with Stochastic Demands". International Transactions in Operational Research doi:10.1111/itor.12540.

Ibrahim, A. T., and F. L. Hall. 1994. "Effect of Adverse Weather Conditions on Speed-Flow-Occupancy Relationships". Transportation Research Record (1457):184-191.

IPCC 2014, November. "Climate Change 2014”. Synthesis report 1, Intergovernmental Panel On Climate Change.

Jaillet, P., and M. R. Wagner. 2008. "Online Vehicle Routing Problems: A Survey". In The Vehicle Routing Problem: Latest Advances and New Challenges, edited by B. Golden et al., 221-237. Boston, MA: Springer US.

Juan, A., J. Faulin, S. Grasman, D. Riera, J. Marull, and C. Mendez. 2011a. "Using Safety Stocks and Simulation to Solve the Vehicle Routing Problem with Stochastic Demands". Transportation Research Part C: Emerging Technologies 19(5):751-765.

Juan, A. A., J. Faulin, J. Jorba, D. Riera, D. Masip, and B. Barrios. 2011b. "On the Use of Monte Carlo Simulation, Cache and Splitting Techniques to Improve the Clarke and Wright Savings Heuristics". Journal of the Operational Research Society 62(6):1085-1097.

Juan, A. A., J. Faulin, J. Jorba, J. Caceres, and J. M. Marquès. 2013. "Using Parallel \& Distributed Computing for Real-Time solving of Vehicle Routing Problems with Stochastic Demands". Annals of Operations Research 207(1):43-65.

Juan, A. A., B. B. Barrios, E. Vallada, D. Riera, and J. Jorba. 2014a. "A Simheuristic Algorithm for Solving the Permutation Flow Shop Problem with Stochastic Processing Times". Simulation Modelling Practice and Theory 46:101-117.

Juan, A. A., S. E. Grasman, J. Caceres, and T. Bektaş. 2014b. "A Simheuristic Algorithm for the Singleperiod Stochastic Inventory-routing Problem with Stock-outs". Simulation Modelling Practice and Theory 46:40-52.

Juan, A. A., J. Faulin, S. E. Grasman, M. Rabe, and G. Figueira. 2015a. "A Review of Simheuristics: Extending Metaheuristics to Deal with Stochastic Combinatorial Optimization Problems". Operations Research Perspectives 2:62-72.

Juan, A. A., I. Pascual, D. Guimarans, and B. Barrios. 2015b. "Combining Biased Randomization with Iterated Local Search for Solving the Multidepot Vehicle Routing Problem". International Transactions in Operational Research 22(4):647-667.

Kaparias, I., M. G. Bell, and H. Belzner. 2008. "A New Measure of Travel Time Reliability for In-Vehicle Navigation Systems”. Journal of Intelligent Transportation Systems 12(4):202-211. 
Lamm, R., E. M. Choueiri, and T. Mailaender. 1990. "Comparison of Operating Speeds on Dry and Wet Pavements of Two-Lane Rural Highways". Transportation Research Record (1280):199-207.

Lourenço, H. R., O. C. Martin, and T. Stützle. 2010. "Iterated Local Search: Framework and Applications". In Handbook of Metaheuristics, edited by M. Gendreau and J.-Y. Potvin, 363-397. Boston, MA: Springer US.

Maze, T. H., M. Agarwai, and G. Burchett. 2006. "Whether Weather Matters to Traffic Demand, Traffic Safety, and Traffic Operations and Flow". Transportation research record: Journal of the transportation research board 1948(1):170-176.

Nogal, M., A. O'Connor, B. Caulfield, and W. Brazil. 2016. “A Multidisciplinary Approach for Risk Analysis of Infrastructure Networks in Response to Extreme Weather". Transportation Research Procedia 14:7885.

Nogal, M., A. O'Connor, P. Groenemeijer, M. Luskova, M. Halat, J. Clarke, P. Van Gelder, and K. Gavin. 2018. "Assessment of the Impacts of Extreme Weather Events Upon the Pan-European Infrastructure to the Optimal Mitigation of the Consequences". Transportation Research Procedia:in press.

Pagès-Bernaus, A., H. Ramalhinho, A. A. Juan, and L. Calvet. 2017. "Designing E-Commerce Supply Chains: A Stochastic Facility-Location Approach". International Transactions in Operational Research doi:10.1111/itor.12433.

Panadero, J., J. Doering, R. Kizys, A. A. Juan, and A. Fito. 2018. "A Variable Neighborhood Search Simheuristic for Project Portfolio Selection Under Uncertainty". Journal of Heuristics doi:10.1007/s10732-0189367-z.

Rakha, H., M. Farzaneh, M. Arafeh, and E. Sterzin. 2008. "Inclement Weather Impacts on Freeway Traffic Stream Behavior". Transportation Research Record: Journal of the Transportation Research Board 2071(1):8-18.

Rakha, H., I. El-Shawarby, and M. Arafeh. 2010. "Trip Travel-Time Reliability: Issues and Proposed Solutions”. Journal of Intelligent Transportation Systems 14(4):232-250.

Spiess, H. 1990. "Technical Note-Conical Volume-Delay Functions”. Transportation Science 24(2):153158.

Tagliapietra, S., and G. Zachmann. 2018. “Addressing Europe's Failure to Clean Up the Transport Sector". Technical Report 2, Bruegel.

Tatarakis, A., and I. Minis. 2009. "Stochastic Single Vehicle Routing with a Predefined Customer Sequence and Multiple Depot Returns". European Journal of Operational Research 197(2):557-571.

Tillman, F. A. 1969. "The Multiple Terminal Delivery Problem with Probabilistic Demands". Transportation Science 3(3):192-204.

United States. 1964. Traffic Assignment Manual for Application with a Large, High Speed Computer. Washington, U.S. Dept. of Commerce, Bureau of Public Roads, Office of Planning, Urban Planning Division; for sale by the Superintendent of Documents, U.S. Govt. Print. Off.

Zuhori, S. T., Z. J. Peya, and F. Mahmud. 2012. "A Novel Three-Phase Approach for Solving Multi-Depot Vehicle Routing Problem with Stochastic Demand”. Algorithms Research 1(4):15-19.

\section{AUTHOR BIOGRAPHIES}

ALEJANDRO ESTRADA-MORENO is a postdoc in the Computer Science, Multimedia and Telecommunication Department at the Open University of Catalonia. He is also a researcher of the ICSO research group at IN3. He holds a Ph.D. in Computer Engineering and Mathematics from Rovira i Virgili University and a M.Sc. in Mathematics and a B.Sc. in Computer Science from Havana University. His main research interests have focused on Graph Theory and Combinatorics, Analytics and Operations Research, Simheuristics, Logistics and Transportation. He has published 14 articles in JCR-indexed journals, and four documents indexed in Scopus. His email email address is aestradamo@uoc.edu. 
CARLES SERRAT is an Associate Professor at the Department of Mathematics at the Universitat Politècnica de Catalunya-BarcelonaTECH, at the Barcelona School of Building Construction (Catalonia, Spain). His areas or research include, but are not limited to, methodological and applied statistics as well as methaheuristics to fields like public health, construction, civil engineering, economy, logistics, and transport. Specifically, he focuses on approaches based on survival analysis techniques, longitudinal data analysis, and missing data analysis. Professor Serrat has been granted for visiting scholarships at Harvard University and Hasselt University and visiting researcher stays at Open University of Catalonia and Trinity College Dublin. His website address is http://cserrat.wordpress.com/ and his email address is carles.serrat@upc.edu.

MARIA NOGAL, Ph.D, MSc, MRes, is an Assistant Professor at the Trinity College Dublin (Ireland). Her expertise includes resilience of interconnected systems, involving land transport networks and their critical infrastructure, and transport modelling. She is also the Principal Investigator of TrinityHaus-School of Engineering. She has published over 60 peer-reviewed scientific papers, and has been awarded with the International Abertis Prize and the XIV Talgo Award. Her email address is nogalm@tcd.ie.

MARTA CAVERO-LAZARO is in her last year at Universitat Autònoma de Barcelona and will graduate with a double degree in Mathematics and Physics. Currently, she is doing an internship at the Open University of Catalonia at the ICSO research group at the IN3. She is taking part in some projects related to optimization, operational research, and data analysis. Her email address is mcaverola@uoc.edu

ANGEL A. JUAN is Professor of Operations Research \& Industrial Engineering in the Computer Science Dept. at the Universitat Oberta de Catalunya (Spain). He is also the coordinator of the ICSO research group at the IN3. Dr. Juan holds a Ph.D. in Industrial Engineering and an M.S. in Mathematics. He completed a predoctoral internship at Harvard University and postdoctoral internships at Massachusetts Institute of Technology and Georgia Institute of Technology. His main research interests include applied optimization and simulation (metaheuristics and simheuristics) in computational transportation and logistics, Internet computing, and computational finance. He has published more than 65 articles in JCR-indexed journals and more than 165 documents indexed in Scopus. His website address is http://ajuanp.wordpress.com and his email address is ajuanp@uoc.edu. 